\title{
Helga Einsele - Ein Leben um der Überzeugung willen
}

A m 13. Februar 2005 ist Helga Einsele im Alter von 94 Jahren in Frankfurt a.M. verstorben. Am 21. Februar 2005 nahmen in einer eindrucksvollen Feierstunde ihre Familie, Vertreter des deutschen und internationalen Strafvollzugs, der Universität Frankfurt, frühere Mitarbeiterinnen und Mitarbeiter aus der Frauenanstalt und Freunde und Begleiter ihres Lebensweges Abschied von dieser Frau, die sie alle in einmaliger Art und Weise beindruckt und geprägt hatte.

"Schwimmen gegen den Strom - um der Überzeugung willen »- so lautet der Titel der Festschrift zu ihrem 80ten Geburtstag. Ein Menschenbild der positiven Zuwendung, eine unbeirrbare politische Ethik, die Suche nach etwas Besserem als der Strafe - dies waren die theorie- und handlungsleitenden Merkmale, die ihren Lebensweg in unvergleichbarer Art kennzeichnen. Gerade in Zeiten des kriminalpolitischen Umbruchs wird deutlich, welch ein Mangel an derartig glaubwürdigen, überzeugten und überzeugenden Persönlichkeiten eingetreten ist.

Helga Einsele war Zeit ihres Lebens - und bis ins hohe Alter - eine politisch aktive Frau. Sie setzte sich schon zu ihren Schulzeiten ein gegen Ungerechtigkeit und Unterdrückung. Geprägt durch ein politisches Elternhaus und durch die Frauenbewegung zeichnete sie ein Leben lang ihre »liebenswürdige Widersetzlichkeit« aus. Sie strebte zusammen mit Vertrauten und Freunden grundlegende Reformen an - sie lebte und praktizierte aber auch im Alltag die kleinen Schritte, die sie - liebenswürdig und unerbittlich zugleich beharrlich und mit grosser Überzeugungskraft durchsetzte.

Helga Einsele hat es immer im Sinne ihres Lebensauftrages verstanden, persönliche und interdisziplinäre Netzwerke herzustellen und zu pflegen. Ihr kritischer und wacher Geist und ihre warmherzige persönliche Zuwendung beeindruckten und bereicherten alle, die mit ihr Kontakt hatten. Eine Trennung zwischen Privatem und Dienstlichem, zwischen Politischem und Unpolitischem, zwischen gefangenen und freien Menschen gab es für sie nicht - sie dachte und handelte vernetzt und in Zusammenhängen.

Für diesen Anspruch an sich selbst und andere zahlte sie einen hohen Preis - »sie war immer im Dienst « - im Dienst ihrer Sache, ihres Lebensauftrages, ihrer Überzeugung. So erzielte sie zwar ein Höchstmass an Wirkung, wurde aber auch angefeindet, ausgegrenzt und ausgeschlossen aus politischen Gremien und Entscheidungsprozessen

So war sie nie mainstream, wollte es auch nicht werden. Sie brauchte die Widersetzlichkeit als Strategie zum Überleben und zur Verteidigung ihrer Unabhängigkeit. Tiefe und Radikalität einer unbeirrbaren politischen Ethik und absolute Glaubwürdigkeit im persönlichen Handeln kennzeichnen ihr Lebenswerk und ihr Leben , sind ihr Vermächtsnis, ihr Auftrag und ihre Herausforderung an die Nachfolger.

\section{Helga Einsele als Anstaltsleiterin}

Vieles, was heute zu den Standards in deutschen Gefängnissen zählt, hat Helga Einsele bereits in den frühen Jahren ihrer Tätigkeit als Anstaltsleiterin vorweggenommen. In der militärisch organisierten Frauenhaftanstalt, in der die gefangenen Frauen noch mit verschränkten Händen auf dem Rücken zum Morgenappell antreten mussten, hat sie schon in den 50-er Jahren den Gruppenvollzug und die soziale Gruppenarbeit, geleitet durch je eine Fürsorgerin, eingeführt. Es folgten die ersten Ansätze für qualifizierte Ausbildungsabschlüsse vor der Industrie- und Handelskammer, die später zu einem bundesweit anerkannten Modellprojekt wurden. In den 60-er Jahren führte sie eine Gefangenenmitverantwortung ein und ein Sprecherinnensystem. Die ersten Gefangenenzeitungen entstanden aus dieser Initiative heraus. Ebenfalls öffnete sie die Anstalt für Ehrenamtliche, orientiert an dem englischen System der prison visitors und beteiligte diese schon bald an der Bildungs- und Gruppenarbeit im Vollzug. Weit vor Inkrafttreten des Strafvollzugsgesetzes führte sie ein Konferenzsystem in der Anstalt ein, außerdem eine Zugangsstation für die ersten Wochen der Inhaftierung, in der durch die Pschyologin und die Sozialarbeiterin dieser Station eine umfassende Anamnese für die spätere Vollzugsplanungskonferenz erarbeitet wurde. Weitere Reformen betrafen die Vollzugsplanung, den offenen Vollzug und Freigang sowie eine gründliche und fundierte Entlassungsvorbereitung. Sie professionalisierte den interdisziplinären "Sozialdienst«, indem sie die täglichen Teambesprechungen, die sogenannten »Mittagsrunden « einführte und diese nach Einführung der 5-Tage-Woche eisern auch an den Samstagen einberief.

Ein besonderes Anliegen war ihr die Vermeidung der Trennung von inhaftierten Müttern von ihren neugeborenen Kindern. Bereits Ende der 50er Jahre ermöglichte sie durch ausgedehnte Stillphasen den Verbleib der neugeborenen Kinder bei ihren Müttern im Anstaltskrankenhaus. Aus diesen Anfängen heraus ist später das erste Mutter-Kind-Heim im Strafvollzug entstanden und die Regelung im Strafvollzugsgesetz, in Frauenanstalten Mutter-Kind-Einrichtungen vorzusehen. Ebenso wie die gemeinsame Unterbringung von Müttern und Kindern in Haft war ihr eine qualifizierte Nachbetreuung der Frauen nach der Entlassung ein großes Anliegen. Dass diese Nachbetreuung um so effektiver ist, je früher sie bereits im Vollzug beginnt, motivierte sie nach ihrer Pensionierung dazu, zusammen mit anderen die Anlaufstelle für straffällig gewordene Frauen ins Leben zu rufen und sie wissenschaftlich zu begleiten. Die Anlaufstelle ist mittlerweile fest etabliert und wurde von Helga Einsele bis $\mathrm{zu}$ ihrem Tod begleitet und unterstützt.

Helga Einsele - Vita
geboren 1910 in Döllau bei
Halle
aufgewachsen in Torgau und
Lüneburg
seit 1929 Studium der Rechts-
wissenschaften in Königsberg,
Breslau und Heidelberg
1931/1932 Studium in den USA
sowie praktische soziale Arbeit
bei der weiblichen Polizei New
York
1939 Promotion bei Gustav
Radbruch über »Das Frauen-
gericht in New York«
1939 bis 1947 Leben und Arbei-
ten mit dem Ehemann in Österreich
1941 Geburt der Tochter Nele
1. 11. 1947 auf Empfehlung von Gustav Radbruch zur Leiterin der
Frauenhaftanstalt Frankfurt am Main (Preungesheim) ernannt
1967 Mitgl. der Stravollzugskommission des Bundesjustizministriums
1969 Fritz-Bauer-Preis der Humanistischen Union
1974 Beccaria-Medaillle in Gold von der Deutschen Kriminologischen
Gesellschaft
1975 Verabschiedung in den Ruhestand
1975 Honorarprofessorin an der Johann-Wolfgang-Goethe Universität
Frankfurt a.M.
1976 Humanitärer Preis der Deutschen Freimaurer
1979 Wilhelm-Leuschner-Medaille
1982 Geburt des Enkels David
Biogrstorben in Frankfurt a.M.

